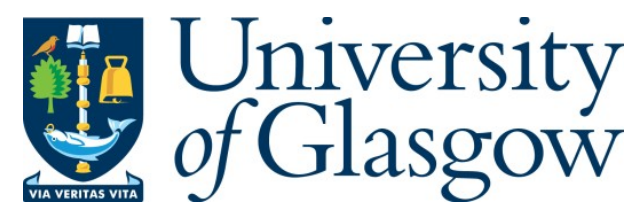

Chen, G., Wang, Y., Tang, L., Wang, K. and Yu, Z. (2020) Large eddy simulation of thermally induced oscillatory flow in a thermoacoustic engine. Applied Energy, 276, 115458.

(doi: 10.1016/j.apenergy.2020.115458)

This is the Author Accepted Manuscript.

There may be differences between this version and the published version. You are advised to consult the publisher's version if you wish to cite from it.

https://eprints.gla.ac.uk/219206/

Deposited on: 29 June 2020

Enlighten - Research publications by members of the University of Glasgow http://eprints.gla.ac.uk 


\title{
Large eddy simulation of thermally induced oscillatory flow in a thermoacoustic engine ${ }^{\dagger}$
}

\author{
Geng Chen ${ }^{1,2}$, Yufan Wang ${ }^{3}$, Lihua Tang1, ${ }^{1, *}$ Kai Wang ${ }^{4}$, Zhibin Yu ${ }^{2}$
}

1. Department of Mechanical Engineering, University of Auckland, 20 Symonds Street, Auckland 1010, New Zealand

2. James Watt School of Engineering, University of Glasgow, Glasgow G12 8QQ, UK

3. Institute of Railway Transit, Tongji University, Shanghai 201800, China

4. Institute of Refrigeration and Cryogenics, College of Energy Engineering, Zhejiang University, Hangzhou 310027, China

\begin{abstract}
In this paper, a comprehensive high-fidelity three-dimensional computational fluid dynamic research using large eddy simulation has been conducted to investigate a standing-wave quarter-wavelength thermoacoustic engine that consists of a hot buffer, a stack and a resonator. The performance of the thermoacoustic engine has been analysed in four aspects. Firstly, the dynamic characteristics of the engine during the initial start-up process are investigated when changing the temperature gradient imposed on the stack. Numerical results are compared with those from a system-wide reduced-order network model based on linear thermoacoustic theory. Secondly, the acoustic behaviour of the engine operating at steady state is studied. Fourier Series Model is utilized to decompose the steady-state acoustic pressure oscillations which reveals the unstable longitudinal acoustic modes excited in the engine. The stack serves as an energy source for the fundamental mode while it extracts acoustic power from the second harmonic. Thirdly, the hydrodynamic performances of the engine are inspected, and the obtained three-dimensional flow fields inside the engine enable us to probe into rich nonlinear phenomena including minor losses, mass streaming, etc. Finally, the heat transfer characteristics have been analysed by examining the mean temperature field and transversal heat fluxes along the engine. This research demonstrates that the large eddy simulation framework is effective in simulating the thermally induced oscillatory flow inside thermoacoustic engines. The multi-perspective analytical methodologies are valuable in comprehending the engine performance and provide guidelines for the design and optimization of efficient thermoacoustic engines for recovering waste thermal energy from various sources.
\end{abstract}

Keywords: Thermoacoustic engine; Large eddy simulation; Network model; Minor losses; Mass streaming;

\footnotetext{
$\dagger$ The short version of the paper was presented at ICAE2019, Aug 12-15, Västerås, Sweden. This paper is a substantial extension of the short version of the conference paper.
} 


\begin{tabular}{|c|c|c|c|}
\hline \multicolumn{4}{|c|}{ Nomenclature } \\
\hline$A$ & cross-section area $\left(\mathrm{m}^{2}\right)$ & $\varepsilon$ & dissipation rate \\
\hline$a$ & sound speed $(\mathrm{m} / \mathrm{s})$ & $\theta$ & phase difference \\
\hline$c_{p}$ & isobaric specific heat $(\mathrm{J} / \mathrm{kgK})$ & $\kappa$ & thermal conductivity of fluid (W/mK) \\
\hline$D$ & diameter of resonator $(\mathrm{m})$ & $\mu$ & dynamic viscosity $(\mathrm{kg} / \mathrm{sm})$ \\
\hline$d$ & gaps between stack plates $(\mathrm{m})$ & $v$ & kinematic viscosity $\left(\mathrm{m}^{2} / \mathrm{s}\right)$ \\
\hline$e_{t}$ & total energy per unit mass $(\mathrm{J} / \mathrm{kg})$ & $\xi$ & displacement $(\mathrm{m})$ \\
\hline$f$ & oscillation frequency $(\mathrm{Hz})$ & $\rho$ & density $\left(\mathrm{kg} / \mathrm{m}^{3}\right)$ \\
\hline$f_{\alpha, v}$ & thermo-viscous functions & $\sigma$ & Prandtl number \\
\hline$g$ & thermoacoustic source term & $\tau$ & relaxation time (s) \\
\hline$h$ & specific enthalpy & $\omega$ & angular frequency $(\mathrm{rad} / \mathrm{s})$ \\
\hline$j$ & $\sqrt{-1}$ & & \\
\hline$p$ & pressure $(\mathrm{Pa})$ & \multicolumn{2}{|c|}{ Subscripts } \\
\hline$Q$ & heat flow (W) & 0 & mean values \\
\hline$q$ & heat flux $\left(\mathrm{W} / \mathrm{m}^{2}\right)$ & 1 & complex quantity \\
\hline $\operatorname{Re}_{\delta}$ & local Reynolds number & $\alpha$ & thermal effects \\
\hline$S_{t}$ & Stokes number & $v$ & viscous effects \\
\hline$s$ & entropy $(\mathrm{J} / \mathrm{K})$ & & \\
\hline$T$ & temperature $(\mathrm{K})$ & \multicolumn{2}{|c|}{ Abbreviations } \\
\hline$t$ & time $(\mathrm{s})$ & $\mathfrak{R}[]$ & real part of a complex quantity \\
\hline$t_{s}$ & stack plate thickness (m) & $\mathfrak{I}[]$ & imaginary part of a complex quantity \\
\hline$U$ & volume velocity $\left(\mathrm{m}^{3} / \mathrm{s}\right)$ & CFD & computational fluid dynamics \\
\hline \multirow[t]{2}{*}{$Z$} & impedance per unit mass $\left(\mathrm{kg} / \mathrm{m}^{3} \mathrm{~s}\right)$ & CPUh & $1 \mathrm{CPU} \times 1$ hour \\
\hline & & FFT & Fast Fourier Transform \\
\hline \multicolumn{2}{|c|}{ Greek symbols } & FSM & Fourier Series Model \\
\hline$\alpha$ & thermal diffusivity $\left(\mathrm{m}^{2} / \mathrm{s}\right)$ & LES & large eddy simulation \\
\hline$\gamma$ & ratio of isobaric to isochoric specific heat & HPC & high-performance computing \\
\hline$\delta$ & penetration depth $(\mathrm{m})$ & UDF & User Defined Function \\
\hline
\end{tabular}

\section{Introduction}

Thermoacoustic engines (TAEs) are the acoustical equivalent of their conventional counterparts such as Stirling engines, but have no moving parts $[1,2]$. They use delicately designed acoustic network to force gas parcels within the thermoacoustic core (stack or regenerator) to experience engine cycles in thermodynamics, so that heat is converted to acoustic energy $[3,4]$. The acoustic energy produced within such engines can be further converted into electricity through transducers such as linear alternators, piezoelectric transducers, magnetohydrodynamic generators, bidirectional turbines [5]. 
Hence, TAEs provide a promising approach for recovering low-grade heat such as geothermal energy [6], industrial waste heat [7, 8], solar thermal energy [9] and exhaust heat of internal combustion engines [10].

Rayleigh's criterion [11] qualitatively elucidates the thermoacoustic effect. In the late 1970s, Rott [12] made significant theoretical breakthroughs in the stability analysis of TAEs and developed a linear theory for estimating the stability limit. Swift [13] then improved the linear theory by introducing a total-power equation in addition to Rott's approximation of continuity and momentum equations. The quasi-one-dimensional treatment in Rott and Swift's framework ignores nonlinear terms in fundamental conservative equations. The linear thermoacoustic theory provides a power tool for designing and analysing thermoacoustic devices when the acoustic amplitude is relatively low [14, 15]. However, substantial discrepancies are observed when the TAEs operate with high-amplitude acoustic oscillations, where nonlinear phenomena, such as minor losses, acoustic streaming, turbulence and harmonics occur [16]. Computational fluid dynamic (CFD) simulations of TAEs can then be advantageous since they solve the fully coupled nonlinear fluid-dynamical equations, and therefore are capable of capturing the aforementioned nonlinear effects. Successful applications of CFD techniques on thermoacoustic devices were found in the literature [17-19].

Although numerical simulations via CFD provide an alternative to study TAEs, there are a few areas that still need improving. Firstly, three-dimensional (3-D) simulations of full-scale thermoacoustic devices are still scarce. CFD simulations of TAEs are time-consuming and computationally expensive [20, 21]. To achieve high-resolution simulation results, refined meshes and small time-step sizes are preferred, which contributes to huge computational costs. Most of the previous work used an axisymmetric two-dimensional (2-D) model with a truncated computational domain (either through a reduced number of stack plates or truncation of resonator) to simplify the problem [22]. To address this issue, we establish a 3-D full-scale TAE model and carry out numerical simulations on the platform of high-performance computing (HPC). Secondly, to the authors' best knowledge, there are few reports on large eddy simulation (LES) of self-excited acoustic oscillations inside TAEs. Previous simulation work [23, 24] normally adopted unsteady Reynolds-Averaged Navier-Stokes (URANS) models (e.g., standard $k-\varepsilon$ ) to capture the dynamics of complex flows in TAEs. URANS approach only solves the averaged quantities while the effect of all scales of instantaneous turbulent motion is modelled by a turbulence model. The URANS-based turbulence models are sometimes unreliable due to inherent inability to resolve transient flow features such as laminar to turbulent transition, vortex shedding, turbulence generation and so on [25]. Thereby, this study takes advantages of high-resolution LES methodology to simulate the small-scale, near-wall flows in the TAE by numerically resolving the filtered Navier-Stokes equations in a wide range of time and length scales. Thirdly, a comprehensive multiple-points-of-view description of simulation results is indispensable. Thermoacoustics is an interdisciplinary topic that involves a blend of subjects such as acoustics, 
dynamics, fluid mechanics, heat transfer and thermodynamics [26, 27]. Therefore, this numerical study aims to interpret the thermoacoustic effect and the energy conversion process from broader perspectives.

As discussed above, the key objective of this paper is to investigate the thermally induced oscillatory flow inside the TAE via 3-D LES. This research provides a comprehensive view of the operating characteristics of the TAE by exploring its dynamic, acoustic, hydrodynamic and heat transfer performances. The numerical/analytical results and methodologies in this study will lay the foundation for the control and adjustment of acoustic, flow and temperature fields inside thermoacoustic devices in future studies. The rest of the paper is organized as follows. Section 2 introduces the numerical procedure adopted in this study. Section 3 analyses the dynamic behaviour of a full-scale TAE in the initial start-up process. Section 4 describes the acoustic characteristics of the TAE operating at steady state. Section 5 elaborates on the hydrodynamic performances that deepen the understanding of diverse nonlinear phenomena. Section 6 discusses the heat transfer inside the TAE by examining the temperature filed and heat fluxes. Finally, concluding remarks are drawn in Section 7.

\section{Numerical methodology}

\subsection{Model description}

Figure 1(a) shows the sketch of a full-scale quarter-wavelength standing-wave thermoacoustic engine (TAE) with its left end closed and right end opened to the ambient (no external loads). The TAE is composed of a hot buffer, a resonator and a parallel-plate stack sandwiched between a hot heat exchanger (left side) and an ambient heat exchanger (right side). For simplicity, the heat exchangers are omitted in the model, and instead, a prescribed axial linear temperature profile (from $T_{\mathrm{h}}$ to $T_{\mathrm{a}}$ ) is imposed on the stack plate surfaces. Detailed information of such thermal boundary condition is given in Appendix A. The working fluid in the TAE is dry air initially at $p_{0}=101,325 \mathrm{~Pa}, T_{\mathrm{a}}=300 \mathrm{~K}$. The geometric dimensions of the TAE and thermophysical properties of the working fluid are summarized in Table 1.

Figure 1(b) depicts the three-dimensional (3-D) computational domain. To reduce the computational cost, one quarter of a complete TAE is modelled with periodic boundary conditions specified at the lateral interfaces. The 3-D model is then meshed with high-quality structured grids as shown in Figure 1(c), where solid domains of the stack plates are not meshed. When generating the meshes, special attention is paid to the wall-normal grid sizes near the walls of stack plates, hot buffer and resonator to ensure that they are smaller than the viscous and thermal penetration depths (or Stokes thicknesses) in order to capture the thermoacoustic energy conversion properly. 
(a)

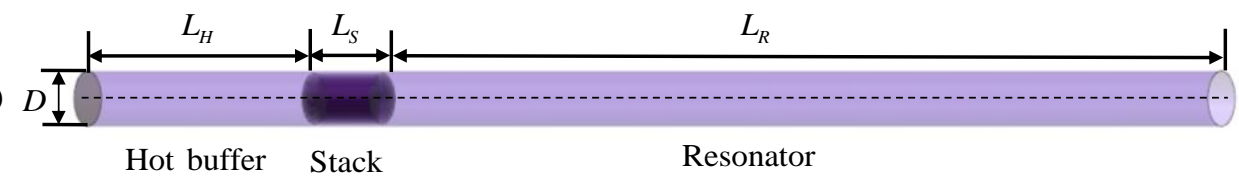

(b)

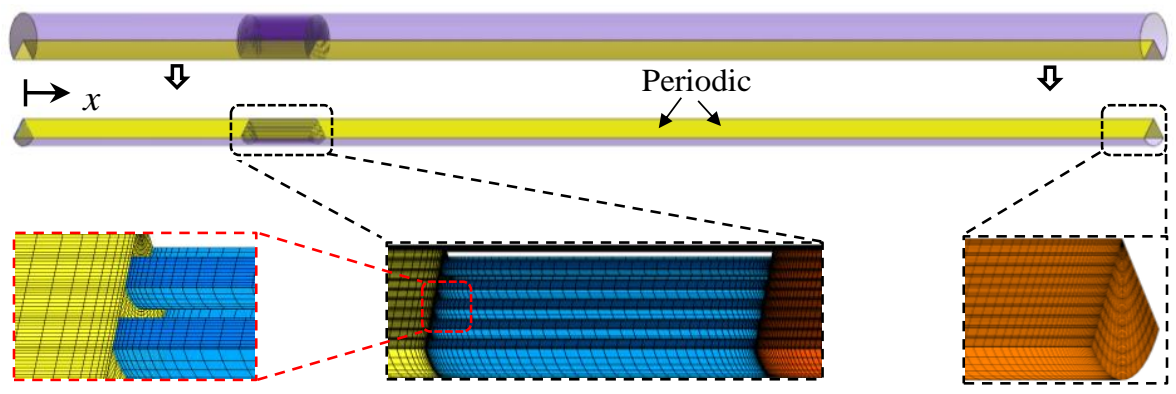

Figure 1. (a) Sketch of a full-scale standing-wave TAE; (b) 3-D computational domain for the TAE;

and (c) close-up views of 3-D meshes of the fluid domains in the hot buffer, stack and resonator.

Table 1. Dimensions of the TAE and thermophysical properties of air at 101,325 Pa, $300 \mathrm{~K}$.

\begin{tabular}{ll}
\hline TAE dimensions & Values \\
\hline Diameter $D$ & $0.021 \mathrm{~m}$ \\
Hot buffer length $L_{\mathrm{H}}$ & $0.1 \mathrm{~m}$ \\
Stack length $L_{\mathrm{S}}$ & $0.03 \mathrm{~m}$ \\
Resonator length $L_{\mathrm{R}}$ & $0.37 \mathrm{~m}$ \\
Stack plate thickness $t_{\mathrm{S}}$ & $0.001 \mathrm{~m}$ \\
Gap between stack plates $d$ & $0.001 \mathrm{~m}$ \\
\hline Air properties & Values \\
Density $\rho_{0}$ & $1.177 \mathrm{~kg} / \mathrm{m}^{3}$ \\
Dynamic viscosity $\mu_{0}$ & $1.847 \times 10^{-5} \mathrm{~kg} /(\mathrm{m} \cdot \mathrm{s})$ \\
Thermal conductivity $\kappa_{0}$ & $0.0263 \mathrm{~W} /(\mathrm{m} \cdot \mathrm{K})$ \\
Speed of sound $a$ & $347 \mathrm{~m} / \mathrm{s}$ \\
Isobaric specific heat $c_{p}$ & $1,006 \mathrm{~J} /(\mathrm{kg} \cdot \mathrm{K})$ \\
Specific heat ratio $\gamma$ & 1.4 \\
\hline
\end{tabular}

\subsection{Simulation procedures}

Figure 2 illustrates the large eddy simulation (LES) procedures adopted in this study which are divided into three steps: pre-processing, iteration process and post-processing. In the pre-processing, a 3-D geometry model is firstly created using a high-end CAD / CAE software package Siemens NX 12.0. This is followed by advanced surface and volume meshing via the meshing tool Altair HyperMesh. 


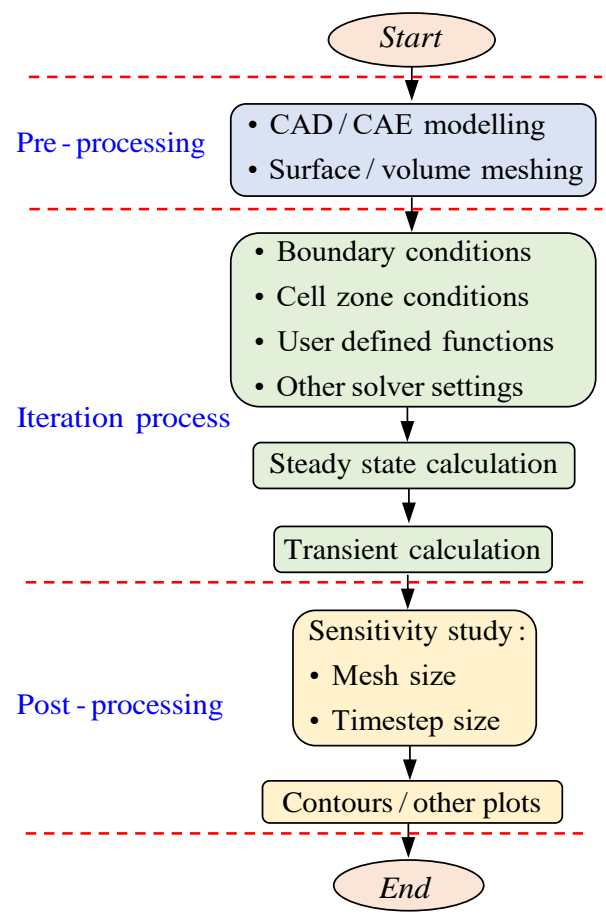

Figure 2. Flow chart of CFD simulations.

In the iteration process, we follow the settings for boundary and cell zone conditions reported in the literature [28]. Non-slip conditions are enforced at all solid walls. The hot buffer and resonator walls are isothermal at $T_{\mathrm{h}}$ and $T_{\mathrm{a}}$ respectively, while linear temperature distributions along the stack walls are specified using a User Defined Function (UDF). The working fluid is assumed to be ideal, satisfying the equation of state $p=\rho R_{g} T$. The time-domain, three-dimensional compressible Navier-Stokes equations in conservation form read

$$
\left\{\begin{array}{l}
\frac{\partial \rho}{\partial t}+\frac{\partial}{\partial x_{i}}\left(\rho u_{i}\right)=0 \\
\rho\left(\frac{\partial u_{i}}{\partial t}+u_{j} \frac{\partial u_{i}}{\partial x_{j}}\right)=\frac{\partial \sigma_{i j}}{\partial x_{j}}-\frac{\partial p}{\partial x_{i}} \\
\frac{\partial}{\partial t}\left(\rho e_{t}\right)+\frac{\partial}{\partial x_{j}}\left(\rho u_{j} e_{t}\right)=-\frac{\partial}{\partial x_{i}}\left(p u_{i}\right)+\frac{\partial}{\partial x_{j}}\left(\sigma_{i j} u_{i}\right)+\frac{\partial}{\partial x_{i}}\left(\kappa \frac{\partial T}{\partial x_{i}}\right)
\end{array}\right.
$$

where $\rho, p, T$ and $e_{t}=e+u_{i}^{2} / 2$ are density, pressure, temperature and total energy per unit mass, respectively. $\sigma_{i j}$ is the viscous stress tensor for Newtonian fluids described by

$$
\sigma_{i j}=2 \mu S_{i j}-\frac{2}{3} \mu \frac{\partial u_{k}}{\partial x_{k}} \delta_{i j}
$$

where $S_{i j}=\left(\partial u_{j} / \partial x_{i}+\partial u_{i} / \partial x_{j}\right) / 2$ is the strain-rate tensor and $\delta_{i j}$ is the Kronecker delta. The subscripts $i, j$ and $k$ are indices of the coordinates. The dynamic viscosity $\mu$ and heat conductivity $\kappa$ are temperature- 
dependent and obey the Sutherland power law [29]. In LES, any variable $\varphi=\bar{\varphi}+\varphi^{\prime}$ is decomposed into large eddies (comparable in size to the characteristic length of the mean flow) which are directly resolved and small eddies (responsible for the dissipation of turbulence kinetic energy) which are modelled empirically [25]. All compressible fluid variables are Favre-filtered (density weighted) so that $\tilde{\varphi}=\overline{\rho \varphi} / \bar{\rho}$. Therefore, Equation (1) after filtering becomes

$$
\left\{\begin{array}{l}
\frac{\partial \bar{\rho}}{\partial t}+\frac{\partial\left(\bar{\rho} \tilde{u}_{i}\right)}{\partial x_{i}}=0 \\
\frac{\partial\left(\bar{\rho} \tilde{u}_{i}\right)}{\partial t}+\frac{\partial\left(\bar{\rho} \tilde{u}_{i} \tilde{u}_{j}\right)}{\partial x_{j}}=\frac{\partial \tilde{\sigma}_{i j}}{\partial x_{j}}-\frac{\partial \bar{p}}{\partial x_{i}}-\frac{\partial \tau_{i j}}{\partial x_{j}} \\
\frac{\partial\left(\bar{\rho} \tilde{e}_{t}\right)}{\partial t}+\frac{\partial\left(\bar{\rho} \tilde{u}_{j} \tilde{e}_{t}\right)}{\partial x_{j}}=-\frac{\partial\left(\bar{p} \tilde{u}_{i}\right)}{\partial x_{i}}+\frac{\partial}{\partial x_{i}}\left(\kappa \frac{\partial \tilde{T}}{\partial x_{i}}\right)+\frac{\partial\left(\tilde{\sigma}_{i j} \tilde{u}_{i}\right)}{\partial x_{j}}+\frac{\partial\left(\tau_{i j} \tilde{u}_{i}\right)}{\partial x_{j}}-\frac{\partial \zeta_{i}}{\partial x_{i}}
\end{array}\right.
$$

where tensors

$$
\tau_{i j}=\bar{\rho}\left(u_{i} u_{j}-\tilde{u}_{i} \tilde{u}_{j}\right) \text { and } \zeta_{i}=\bar{\rho}\left(u_{i} h-\tilde{u}_{i} \tilde{h}\right)
$$

in the filtered momentum and energy equation are modelled by the Wall-Adapting Local Eddy-viscosity (WALE) subgrid-scale (SGS) model [30]. $h=e+p / \rho$ represents the specific enthalpy. The above nonlinear governing equations on the structured grids are resolved in the control-volume-based, finitevolume CFD solver ANSYS Fluent 18.1. The near-wall grid sizes yield wall distance $y$ plus ( $y+$ ) values of around 0.5, which are sufficient for obtaining high-resolution data whilst the maximum CourantFriedrichs-Lewy (CFL) of 0.45 is achieved at the optimal time-step size (Section 2.3) for accurately resolving small eddies [31]. In this study, we implement the following two steps in sequence to simulate the thermally induced oscillatory flow inside the TAE. In step 1, a steady-state simulation is conducted to get converged temperature and pressure fields by imposing a small pressure difference $\left(10^{-3} \mathrm{~Pa}\right)$ across the ends of the TAE. The left end of the TAE is set as pressure inlet while the right end is pressure outlet. A steady-state RANS (Reynolds-Averaged Navier-Stokes) model (i.e., standard $k-\varepsilon$ ) is applied in this step. In step 2, the pressure difference is ceased suddenly (by changing the pressure inlet to a solid wall) which triggers a pressure pulse [28], and a transient simulation using LES is performed thereafter. Besides, transient simulations using the URANS (unsteady RANS) model are also performed for comparison (Sections 2.3 and 3.2).

Finally, in the post-processing, a sensitivity study on the grid and time-step sizes is first conducted. Then, based on the contours and plots produced, the dynamic, acoustic, hydrodynamic and heat transfer characteristics of the TAE are analysed and discussed. 


\subsection{Sensitivity study}

3-D high-fidelity simulations of full-scale TAEs are time-consuming, due to a broad range of length scales (from the Stokes thickness of the order of $10^{-4} \mathrm{~m}$ to the total tube length $L$ of the order of $1 \mathrm{~m}$ ) and time scales (from the time-step of the order of $10^{-5} \mathrm{~s}$ to the running time of $1 \mathrm{~s}$ to reach steady state). This section aims to find the optimal grid and time-step sizes so as to produce relatively accurate results at reasonable computational costs.

Figure 3(a) displays the time history of the closed-end space-averaged acoustic pressure $\langle p\rangle=\int p d A / A$, where $A$ is the cross-sectional area. An example case $\left(T_{\mathrm{h}}=550 \mathrm{~K}\right)$ using LES with the number of nodes of 703,010 shows that it takes 5,867 CPUhs $(1 \mathrm{CPUh}=1 \mathrm{CPU} \times 1$ hour $)$ to reach a running time of $1 \mathrm{~s}$. Three consecutive processes are identified from the evolution of acoustic pressure over time: (i) initial start-up where the amplitude of pressure fluctuations increases exponentially. The dynamic behaviour in this process can be predicted by linearized equations, as will be discussed in Section 3; (ii) saturation where the growth rate of pressure amplitude decays; (iii) steady state where the waveforms are limit cycles oscillating at amplitude $P_{A}$ and fundamental frequency $f_{1}$ as shown in Figure 3(b). Herein, the steady-state pressure amplitude $P_{A}$ and frequency $f_{1}$ are used as the key metrics in the sensitivity study.
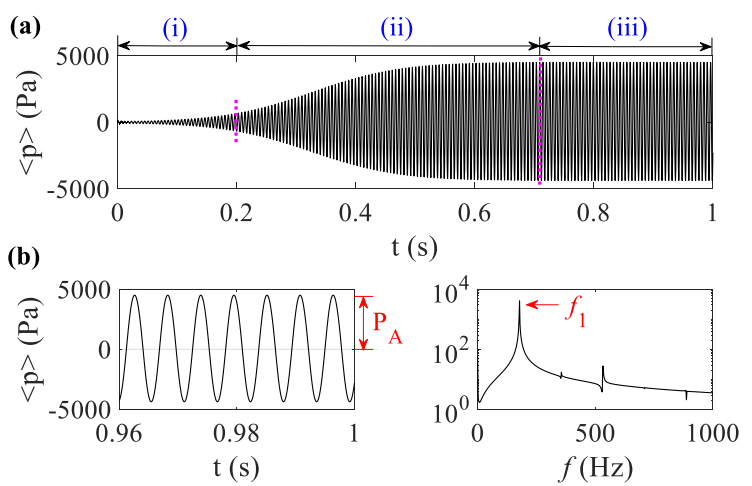

Figure 3. Key metrics in the sensitivity study. (a) Time history of the closed-end, space-averaged acoustic pressure at $T_{\mathrm{h}}=550 \mathrm{~K}$ using the LES model. (b) Steady-state limit cycles.

The effect of grid size on $P_{A}$ and $f_{1}$ is first investigated by refining the mesh in the stack flow channels gradually. In this case, the number of nodes increases from 152,410 to 771,010 and a time-step of $10^{-5}$ $\mathrm{s}$ is used. It is found that, for both LES and URANS models, when the number of nodes increases, $P_{A}$ and $f_{1}$ first decrease, then asymptote and finally become less sensitive. However, the CPUhs per second (CPUhs/sec) increases almost linearly with the number of nodes. Similarly, the effect of time-step on $P_{A}$ and $f_{1}$ is studied by reducing the time-step from $2 \times 10^{-5} \mathrm{~s}$ to $0.8 \times 10^{-5} \mathrm{~s}$ gradually. In this case, the optimal numbers of nodes are used. It is found that, for both LES and URANS models, when the timestep decreases, $P_{A}$ and $f_{1}$ first increase, then asymptote and finally become less sensitive. However, CPUhs/sec seems to increase exponentially as the time-step decreases. Therefore, to compromise the simulation accuracy and computational cost, the optimal numbers of nodes for the LES and URANS 
models are selected as 703,010 and 497,210 respectively, and the optimal time-steps are both chosen at $1 \times 10^{-5} \mathrm{~s}$. The optimal numbers of nodes and time-steps are used in the following calculations.

Table 2 displays $P_{A}$ and $f_{1}$ at different $T_{\mathrm{h}}$ using LES and URANS models at optimal numbers of nodes and time-steps. $f_{1}$ varies no more than $4.5 \mathrm{~Hz}$ between LES and URANS whereas $P_{A}$ is highly affected, being higher for LES. Currently, we are unable to prove the superiority of LES over URANS in terms of $P_{A}$ and $f_{1}$ due to lack of a robust nonlinear, time-domain, theoretical model to predict the limit-cycle behaviour. Previous attempts of building such a model were reported by Wang [32], however, nonlinearity was only accounted for in the regenerator based on an empirical correlation. Improvements will be made in the future by incorporating nonlinear terms based on contributions of nonlinear losses caused by large-amplitude oscillations at steady state.

Table 2. $P_{A}$ and $f$ at $T_{\mathrm{h}}=550,600,650$ and $700 \mathrm{~K}$ using LES and URANS models.

\begin{tabular}{ccccc}
\hline & $T_{\mathrm{h}}=550 \mathrm{~K}$, & $T_{\mathrm{h}}=600 \mathrm{~K}$, & $T_{\mathrm{h}}=650 \mathrm{~K}$, & $T_{\mathrm{h}}=700 \mathrm{~K}$, \\
& $P_{A}(\mathrm{~Pa}), f_{1}(\mathrm{~Hz})$ & $P_{A}(\mathrm{~Pa}), f_{1}(\mathrm{~Hz})$ & $P_{A}(\mathrm{~Pa}), f_{1}(\mathrm{~Hz})$ & $P_{A}(\mathrm{~Pa}), f_{1}(\mathrm{~Hz})$ \\
\hline LES & $4516.6,177.62$ & $6096.3,177.94$ & $7303.0,178.57$ & $8297.1,179.21$ \\
URANS & $3690.3,179.53$ & $5274.3,180.83$ & $6493.0,182.48$ & $7474.8,183.49$ \\
\hline
\end{tabular}

\section{Dynamic characteristics}

This section focuses on the dynamic responses of a full-scale TAE at different temperature gradients. Special attention is given to the transient behaviour of acoustic pressure in the initial start-up process. Theoretical and numerical methodologies are adopted in this study to predict such behaviour.

\subsection{Theoretical analysis}

Equation (1) is difficult to solve due to nonlinearity. To bring down the complications, we adopt the perturbation techniques herein. Formally, each fluid variable, e.g., pressure $p$, can be written in the form of $p=p_{0}+p^{\prime}+p^{\prime \prime}+\cdots$, where the subscript 0 denotes a basic state, such as steady-state equilibrium, superscripts ' and " denote first-order and second-order perturbations, respectively. Substituting these perturbation-form variables into Equations (1), neglecting the second and higher order terms, we have

$$
\left\{\begin{array}{l}
\frac{\partial \rho^{\prime}}{\partial t}+\rho_{0} \nabla \cdot \boldsymbol{u}^{\prime}=0 \\
\rho_{0} \frac{\partial \boldsymbol{u}^{\prime}}{\partial t}=-\nabla p^{\prime}+\mu \nabla^{2} \boldsymbol{u}^{\prime}+\frac{1}{3} \mu \nabla\left(\nabla \cdot \boldsymbol{u}^{\prime}\right) \\
\rho_{0} T_{0} \frac{\partial s^{\prime}}{\partial t}=\kappa \nabla^{2} T^{\prime}
\end{array}\right.
$$

For an ideal gas, the pressure $p$, density $\rho$, temperature $T$ and specific entropy $s$ satisfy

$$
\frac{\rho^{\prime}}{\rho_{0}}=\frac{p^{\prime}}{\rho_{0} a^{2}}-\frac{s^{\prime}}{c_{p}}, \quad \frac{T^{\prime}}{T_{0}}=(\gamma-1) \frac{p^{\prime}}{\rho_{0} a^{2}}+\frac{s^{\prime}}{c_{p}}
$$


where $c_{p}$ is the isobaric specific heat, $\gamma$ is the specific heat ratio and $a$ is the adiabatic sound speed. Substituting the momentum equation into the continuity equation in Equation (5), we have the inhomogeneous acoustic wave equation in thermoacoustics, i.e.,

$$
-\frac{\partial^{2} p^{\prime}}{\partial t^{2}}+a^{2} \nabla^{2} p^{\prime}+\left(\frac{1}{3} v+(\gamma-1) \alpha\right) \frac{\partial}{\partial t} \nabla^{2} p^{\prime}=\frac{\rho_{0} a^{2}}{c_{p}}\left(\frac{1}{3} v-\alpha\right) \frac{\partial}{\partial t} \nabla^{2} s^{\prime}
$$

where $v=\mu / \rho$ denotes the kinematic viscosity and $\alpha=\kappa / \rho c_{p}$ is the thermal diffusivity. To describe the evolution of small pressure perturbations, one needs to obtain the exact solution of Equation (7), which seems rather difficult at present. However, one can also characterize the pressure evolution through a complex frequency $\omega=\omega_{R}+j \omega_{I}\left(\omega_{R}\right.$ is the oscillation frequency; $\omega_{I}$ denotes the growth/attenuate rate $)$ in the frequency domain. The derivation of complex frequency requires the formation of an eigenvalue problem, which could be realized through a system-wide reduced-order network model [33-36].

One of the main merits of network modelling is that it can study the dynamic behaviour of the TAE in a system level. In the network model, the TAE is discretized evenly into numerous segments. Uniform thermophysical properties are assumed in each segment with length $\Delta x$. By assuming harmonic time dependence (e.g., $p^{\prime}=p_{1} e^{j \omega t}$ ), the partial differential equations (PDEs) in Equation (5) are transformed into ordinary differential equations (ODEs). Solving the ODEs and space-averaging across the crosssection, Swift [13] derived the linearized continuity and momentum equations in thermoacoustics, which are

$$
\left\{\begin{array}{l}
\frac{d U_{1}}{d x}=-\left(1+(\gamma-1) f_{k}\right) \frac{j \omega A}{\gamma p_{0}} p_{1}+\frac{f_{k}-f_{v}}{(1-\sigma)\left(1-f_{v}\right)} \frac{1}{T_{0}} \frac{d T_{0}}{d x} U_{1}=-Y p_{1}+g U_{1} \\
\frac{d p_{1}}{d x}=-\frac{j \omega \rho_{0}}{A\left(1-f_{v}\right)} U_{1}=-Z U_{1}
\end{array}\right.
$$

where $p_{1}$ and $U_{1}$ are complex notations of acoustic pressure and volume velocity, $f_{k}$ and $f_{v}$ are spaceaveraged thermoviscous functions [37], $\gamma$ and $\sigma$ denote the specific heat ratio and Prandtl number, $Y$ and $g$ stand for the fluid admittance per unit length and thermoacoustic source term, and $Z$ stands for the fluid impedance per unit length. Therefore, $p_{1}$ and $U_{1}$ at two adjacent segments $(i$ and $i+1)$ are related by a four-port transfer matrix $\mathbf{R}(i)$, i.e.,

$$
\left[\begin{array}{c}
p_{1}(i+1) \\
U_{1}(i+1)
\end{array}\right]=\mathbf{R}(i)\left[\begin{array}{c}
p_{1}(i) \\
U_{1}(i)
\end{array}\right]=\left[\begin{array}{cc}
1 & -Z(i) \Delta x \\
-Y(i) \Delta x & g(i) \Delta x+1
\end{array}\right]\left[\begin{array}{c}
p_{1}(i) \\
U_{1}(i)
\end{array}\right]
$$

By assuming continuity of $p_{1}$ and $U_{1}$ at the interfaces of segments, $p_{1}$ and $U_{1}$ at the TAE ends can be related by a total transfer matrix $\mathbf{R}_{\mathbf{T}}$,

$$
\left[\begin{array}{c}
p_{1}(L) \\
U_{1}(L)
\end{array}\right]=\mathbf{R}_{\mathrm{T}}\left[\begin{array}{c}
p_{1}(0) \\
U_{1}(0)
\end{array}\right], \quad \mathbf{R}_{\mathrm{T}}=\mathbf{R}_{\mathbf{R}} \mathbf{R}_{\mathrm{S}} \mathbf{R}_{\mathrm{H}}
$$


where $\mathbf{R}_{\mathbf{H}}, \mathbf{R}_{\mathbf{S}}$ and $\mathbf{R}_{\mathbf{R}}$ are total transfer matrixes for the hot buffer, stack and resonator, respectively. Solving the characteristic Equation (10) numerically with the impedances $Z(0)=p_{1}(0) / U_{1}(0)=\infty$ and $Z(L)=p_{1}(L) / U_{1}(L)=0$ imposed at both ends yields the complex frequency $\omega$ as a function of hot-end temperature $T_{\mathrm{h}}$. Appendix B presents the application of the reduced-order network model in our previous experimental study [35]. The good agreement between the theoretical estimates and experimental measurements ensures the credibility of the network method used in this study.

\subsection{Numerical simulations}

Figure 4 displays the time series of pressure disturbance in the initial start-up process from LES simulations at $T_{\mathrm{h}}=400,480$ and $550 \mathrm{~K}$, respectively. The disturbance is damped at $400 \mathrm{~K}$, marginallymaintained at $480 \mathrm{~K}$ and amplified at $550 \mathrm{~K}$. Hence, the onset temperature $T_{\mathrm{h}}$, onset from LES is around $480 \mathrm{~K}$ which is $36 \mathrm{~K}$ higher than that predicted from the network model when $\omega_{I}$ is zero. Nonlinearities neglected by the linear theory may account for the discrepancy. As seen in Figure 4, at any $T_{\mathrm{h}}$, the oscillation frequency $\omega_{R}$ and attenuation/growth rate $\omega_{I}$ can be obtained numerically through Fast Fourier Transform (FFT) and exponential fits (envelope curves) for the peaks.

Numerical values of $\omega_{R}$ and $\omega_{I}$ are also obtained from URANS simulations. Table 3 summarizes $\omega_{R}$ and $\omega_{I}$ at $T_{\mathrm{h}}=550,600,650$ and $700 \mathrm{~K}$ using LES, URANS and network models. The results indicate that $\omega_{R}$ varies negligibly between LES and URANS. Nonetheless, $\omega_{I}$ differs substantially: $\omega_{I}$ from LES is closer to the theoretical counterpart. In this regard, LES seems to be more accurate than URANS. One possible explanation is that LES captures the small-scale eddies in full detail using the SGS model rather than modelling eddies of all scales using a single turbulence model as in the URANS simulations [25].

(a)
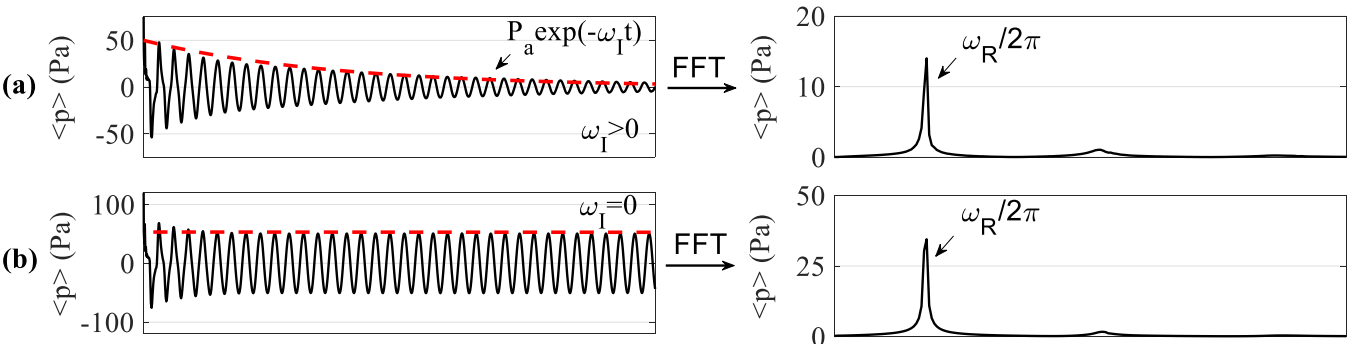

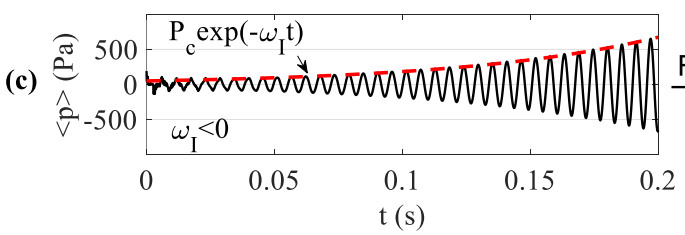

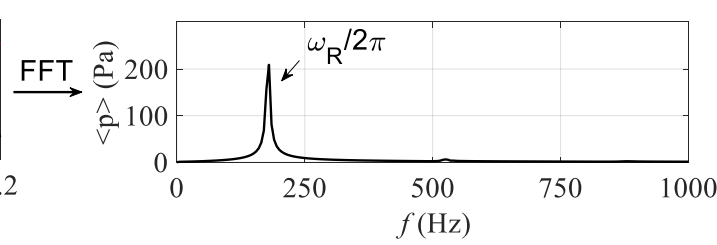

Figure 4. Time series of pressure disturbance in the initial start-up process from LES simulations at:

(a) $T_{\mathrm{h}}=400 \mathrm{~K}$; (b) $T_{\mathrm{h}}=480 \mathrm{~K}$; (c) $T_{\mathrm{h}}=550 \mathrm{~K}$. 
Table 3. Comparison between LES, URANS and network models on $\omega_{R}$ and $\omega_{I}$ at $T_{\mathrm{h}}=550,600,650$ and $700 \mathrm{~K}$.

\begin{tabular}{lllll}
\hline & $T_{\mathrm{h}}=550 \mathrm{~K}$, & $T_{\mathrm{h}}=600 \mathrm{~K}$, & $T_{\mathrm{h}}=650 \mathrm{~K}$, & $T_{\mathrm{h}}=700 \mathrm{~K}$, \\
& $\omega_{R} / 2 \pi, \omega_{I}$ & $\omega_{R} / 2 \pi, \omega_{I}$ & $\omega_{R} / 2 \pi, \omega_{I}$ & $\omega_{R} / 2 \pi, \omega_{I}$ \\
\hline LES & $177.62,-13.05$ & $178.89,-21.21$ & $179.86,-27.37$ & $180.83,-33.27$ \\
URANS & $178.25,-7.41$ & $179.21,-14.27$ & $180.51,-20.40$ & $181.49,-26.22$ \\
Network model & $178.27,-15.38$ & $179.32,-22.28$ & $180.45,-28.73$ & $181.63,-34.63$ \\
\hline
\end{tabular}

\section{Acoustic characteristics}

This section concerns the acoustic behaviour of the TAE operating at steady state. Figure 5 illustrates the decomposition of closed-end, space-averaged pressure oscillations from LES at $T_{\mathrm{h}}=700 \mathrm{~K}$. The Fourier Series Model (FSM) is adopted to decompose the periodic pressure signals. In FSM, the pressure oscillations can be expressed by a sum of cosine functions, so that

$$
\langle p\rangle=\left\langle p_{0}\right\rangle+\sum_{n=1}^{m}\left\langle p_{n}\right\rangle \cos \left(\omega_{n} t\right)
$$

As shown in Figure 5, $\left\langle p_{0}\right\rangle$ exists because of the variation of fluid compressibility in the axial direction along which the mean temperature varies. $\left\langle p_{1}\right\rangle$ stands for acoustic pressure oscillating at the fundament mode, which also dominates the steady-state pressure oscillations. $\left\langle p_{2}\right\rangle$ is the second harmonic, possessing a frequency two times larger than the fundamental one; however, its amplitude is much smaller. Higher harmonics are of no interest and not discussed in this study.

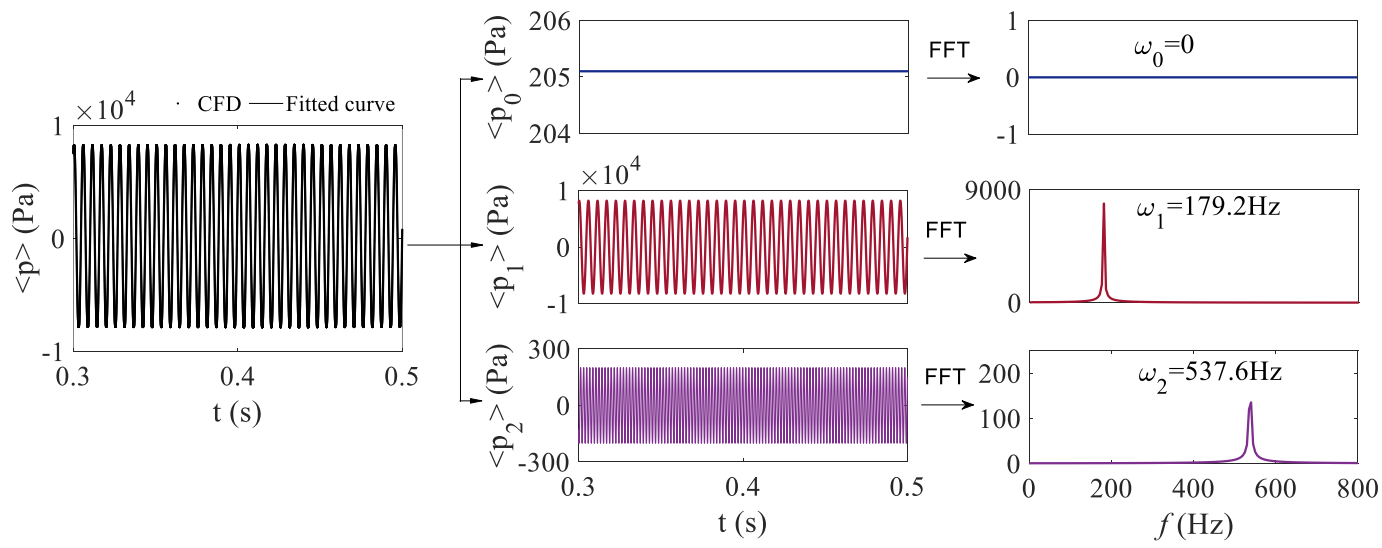

Figure 5. Decomposition of closed-end, space-averaged pressure oscillations at steady state from LES

$$
\text { at } T_{\mathrm{h}}=700 \mathrm{~K} \text {. }
$$

By decomposing the space-averaged pressure and velocity oscillations at different positions in the longitudinal direction, a picture of the longitudinal mode oscillations is generated, as shown in Figure 6. The stack region is shown in the shaded area, in which the velocity increases suddenly due to abrupt 
decrease of cross-sectional area. Standing-wave features are exhibited: the pressure and velocity antinodes locate at the closed end $(x=0)$ and the open end $(x=L)$, respectively. The acoustic power in the figure is described by

$$
I=\sum_{n=1}^{m} I_{2 n}=\sum_{n=1}^{m} \frac{1}{2} A\left\langle p_{n}\right\rangle\left\langle u_{n}\right\rangle \cos \theta_{n}
$$

where $\theta_{n}$ is the phase between the pressure and velocity. In Figure 6(a), acoustic power $I_{2}$ is negative at the left-hand side of the stack but positive on the right-hand side. Therefore, $I_{2}$ flows out of the stack from both sides, which indicates that the stack acts as an acoustic energy source for the fundamental mode. In Figure 6(b), the TAE is three-quarter-wavelength at the second harmonic. The magnitude of acoustic power $I_{4}$ is three orders smaller than $I_{2}$. $I_{4}$ decreases in the stack region which reveals that the stack serves as an acoustic energy sink for the second harmonic. This finding is consistent with the experimental measurements reported by Biwa who attributes the suppression of thermoacoustic shock waves to the decrease of $I_{4}$ in the stack region [38].

(a)
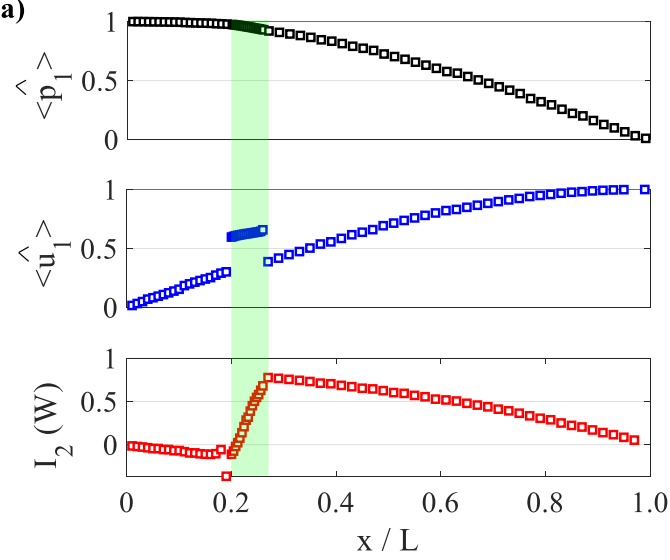

(b)

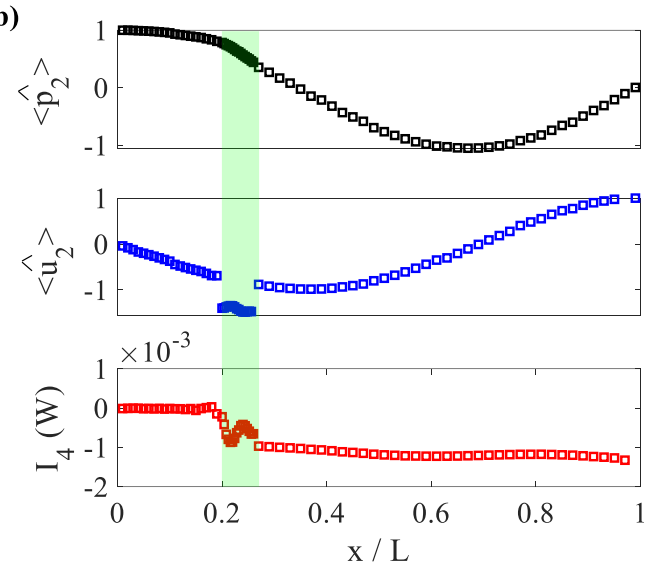

Figure 6. Excited acoustic oscillation modes at $T_{\mathrm{h}}=700 \mathrm{~K}$ from LES. (a) Axial distributions of acoustic pressure, velocity and acoustic power at fundamental frequency; $\left\langle\hat{p}_{1}\right\rangle$ and $\left\langle\hat{u}_{1}\right\rangle$ are normalized pressure and velocity amplitudes at the fundamental mode. (b) Axial distributions of acoustic pressure, velocity and acoustic power at second harmonic; $\left\langle\hat{p}_{2}\right\rangle$ and $\left\langle\hat{u}_{2}\right\rangle$ are normalized pressure and velocity amplitudes at the second harmonic.

\section{Hydrodynamic characteristics}

This section explores the hydrodynamic performances of the TAE operating at steady state. Emphasis is laid on the flow fields inside the TAE which disclose the mechanisms of various nonlinear phenomena reported in the literature. 


\subsection{Momentum diffusion within boundary layers}

Figure 7 displays the instantaneous velocity profiles in one stack channel (at $x=0.115 \mathrm{~m}$ ) and in the resonator (at $x=0.25 \mathrm{~m}$ ) at eight instants $\left(\phi_{1}\right.$ to $\left.\phi_{8}\right)$ during one acoustic cycle at $T_{\mathrm{h}}=700 \mathrm{~K}$ from LES. The velocity profiles can be characterized by the Stokes number,

$$
S_{t}=\sqrt{\omega D_{0}^{2} / v}=\sqrt{2} D_{0} / \delta_{v}
$$

where $D_{0}$ refers to the radius of the pipe or half of the plate gap. $\delta_{v}=\sqrt{2 v / \omega}$ denotes the viscous penetration depth. When the frequency $\omega$ is low, $S_{t}$ is small and $\delta_{v}$ is large. The flow channel is filled up with Stokes layers, leading to velocity profiles similar to a Poisuille flow, as featured by the oscillatory flow in the stack channels $\left(S_{t}=2.71\right)$ in Figure 7(a) from LES. In the high-frequency regime, $S_{t}$ is large and $\delta_{v}$ is small. Only near-wall flow is affected by the viscosity and exhibits the so-called Richardson's annular effects (overshoots), as shown in Figure 7(b) for the oscillatory flow in the resonator $\left(S_{t}=88.97\right)$. These overshoots in the velocity profiles were also reported in an experimental study conducted by Shi et al [39]. Note that the instantaneous velocity profiles in Figure 7 are not symmetric due to nonlinear distortions induced by large-amplitude oscillations and inhomogeneities in the stack region.

The momentum equation in Equation (5) could be further written in the form of a vorticity wave equation

$$
\frac{\partial \boldsymbol{\Omega}}{\partial t}-v \nabla^{2} \boldsymbol{\Omega}=0
$$

where $\boldsymbol{\Omega}=\nabla \times \boldsymbol{u}^{\prime}$. Hence, apart from planar acoustic wave, there is diffusive vorticity wave in the oscillatory flow field. The viscous relaxation time

$$
\tau_{v}=\left(D_{0} / \delta_{v}\right)^{2} / \omega=S_{t}^{2} / 2 \omega
$$

is the characteristic time that determines how fast the vorticity wave propagates. If $D_{0}$ is small or the viscosity $\mu$ is large, $\tau_{v}$ is small. For example, $\tau_{v}=3.26 \times 10^{-3} \mathrm{~s}$ in the stack channel at $x=0.115 \mathrm{~m}$ in Figure 7(a). Then, the flow is dominant by diffusive vorticity wave where the majority of momentum is dissipated irreversibly at a time scale of $\tau_{\nu}$. On the contrary, if $D_{0}$ is large or the viscosity $\mu$ is small, $\tau_{v}$ is large. For instance, $\tau_{v}=3.51 \mathrm{~s}$ in the resonator at $x=0.25 \mathrm{~m}$ in Figure $7(\mathrm{~b})$. In this case, planar acoustic wave dominates transporting momentum reversibly at a time scale of $2 \pi / \omega=5.58 \times 10^{-3} \mathrm{~s}$. 
(a)

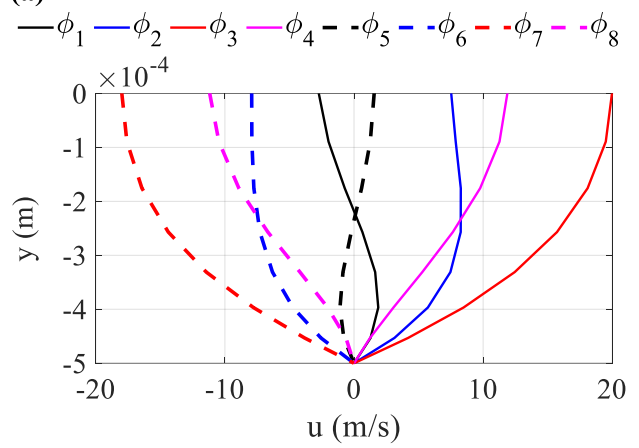

(b)
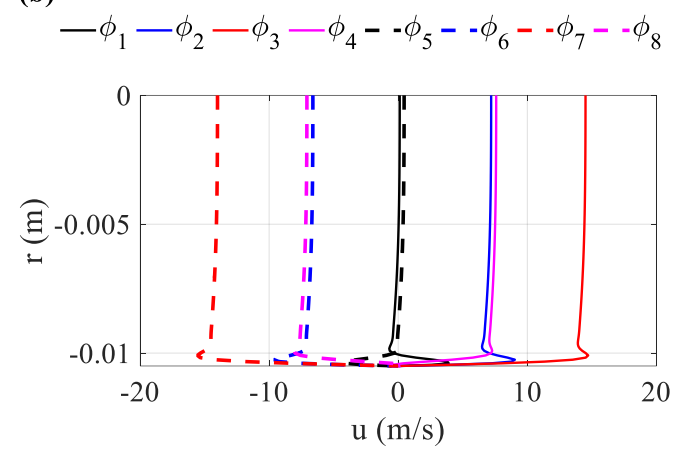

Figure 7. Instantaneous velocity profiles during one acoustic cycle at $T_{\mathrm{h}}=700 \mathrm{~K}$ from LES. (a) Stack channels at $x=0.115 \mathrm{~m}$ where $S_{t}=2.71$ and $\tau_{v}=3.26 \times 10^{-3} \mathrm{~s}$. (b) Resonator at $x=0.25 \mathrm{~m}$ where $S_{t}=$ 88.97 and $\tau_{v}=3.51 \mathrm{~s}$.

\subsection{Minor losses}

Abrupt decrease of cross-sectional area at the stack ends induces vortex shedding in the longitudinal direction that brings about significant minor losses in the TAE. Figure 8 illustrates LES simulation of 3 -D vortex core regions and velocity streamlines around the stack ends at four instants $\left(\phi_{1}, \phi_{3}, \phi_{5}\right.$ and $\phi_{7}$ ) displayed in Figure 7. The method defining the vortex core region is Q-Criterion of level 0.01. The expression of $\mathrm{Q}$ is given by

$$
\mathrm{Q}=\frac{1}{2}\left(\Omega_{i j} \Omega_{j i}-S_{i j} S_{j i}\right)
$$

where $\Omega_{i j}\left(\right.$ or $\left.\Omega_{j i}\right)$ and $S_{i j}\left(\right.$ or $\left.S_{j i}\right)$ are the vorticity-rate and strain-rate tensors, respectively.

At instants $\phi_{1}$ and $\phi_{5}$, the space-averaged velocities approximate zero. Weak toroidal vortex rings are observed at the stack left end, while broken rings and smaller eddies are found at the right end. The right-end velocity streamlines are also disordered because of the turbulence eddies. At instants $\phi_{3}$ and $\phi_{7}$, the space-averaged velocities are the maximum in the opposite directions. The kinetic energy of the flow is high, and strong regular vortex rings are found at a short distance from the stack ends. The velocity streamlines away from the stack ends are straight showing no sign of evident circular flows. Mao [40] experimentally studied the vortex structures at the stack ends using particle image velocimetry (PIV) and displayed their measurements in a 2-D manner. Our 3-D representations of the vortex shedding process at the stack ends share similarities with the PIV measurements and have not been reported by any previous numerical simulations. 

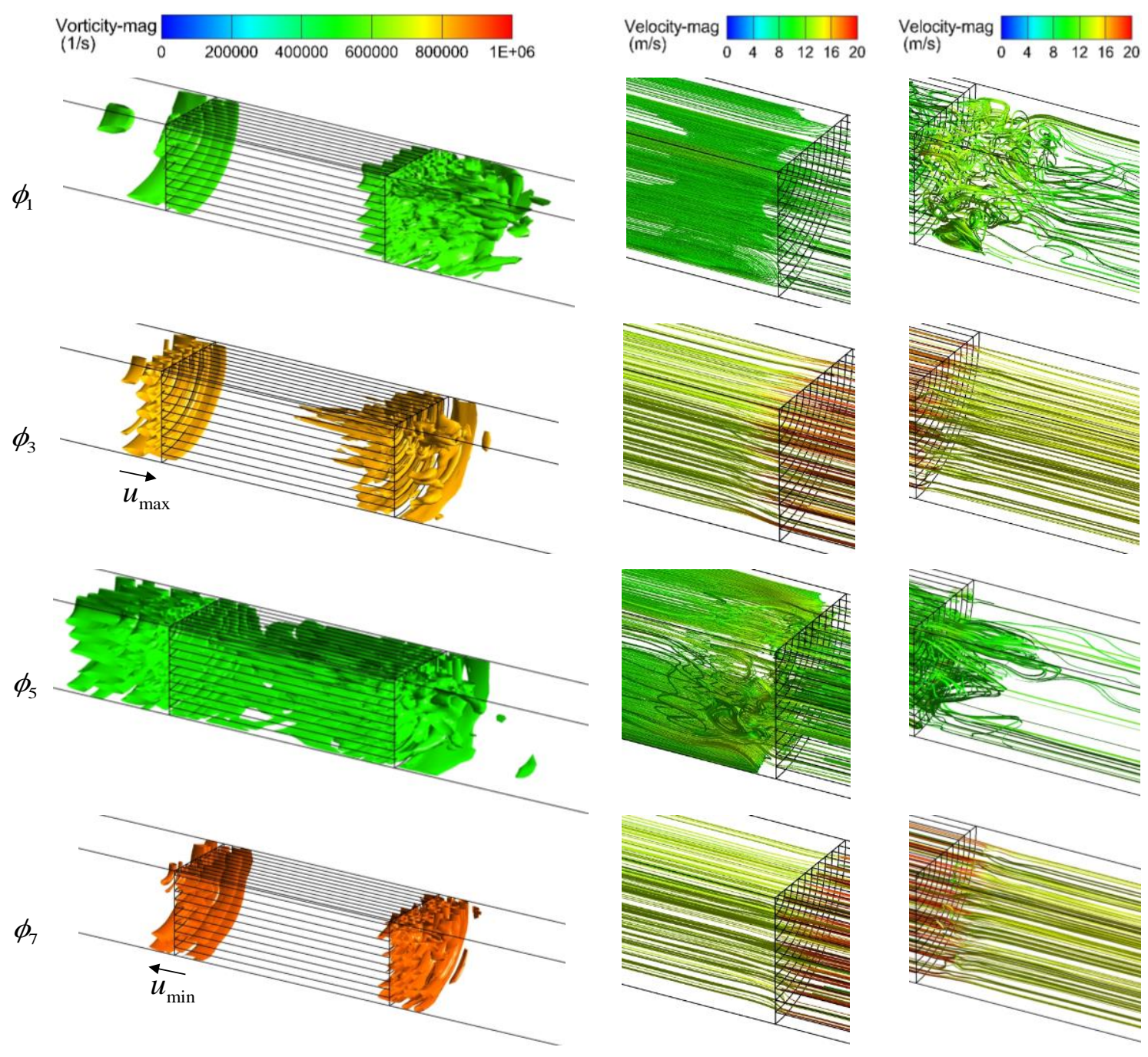

Figure 8. Illustrations of instantaneous vortex core region and velocity streamlines around the stack ends at four different instants at $T_{\mathrm{h}}=700 \mathrm{~K}$ from LES.

\subsection{Mass streaming}

In TAEs, mass streaming refers to a net time-averaged mass flow superimposed on the first-order oscillations. Swift [13] described different types of mass streaming in thermoacoustic devices, including Gedeon streaming [41] that is undesirable in travelling-wave TAEs, Rayleigh streaming [42] driven by boundary-layer effects at the side walls, jet-driven streaming [43] and streaming within a stack/regenerator [44]. We observed the latter three types in this study.

In the simulations, mass streaming can be described by the time-averaged mass flux $\overline{\rho u}$. As shown in Figure 9, the slices of contours of $\overline{\rho u}$ near the stack ends clearly indicate the existence of jet-driven mass streaming that arises from sudden contraction and expansion of the flow channels at the stack ends. Intense jet-driven mass streaming is observed near the stack ends and weakens rapidly in accordance with the breakdown of vortex rings away from the stack ends. 

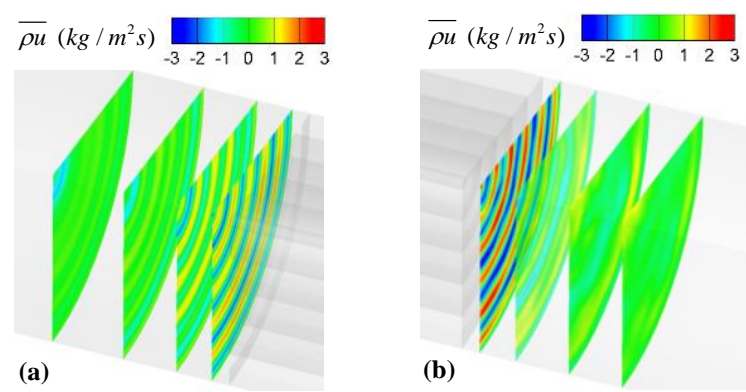

Figure 9. Jet-driven mass streaming at the ends of the stack channels from LES at $T_{\mathrm{h}}=700 \mathrm{~K}$.

Figure 10 shows the values of $\overline{\rho u}$ at selected axial positions in the hot buffer, one representative stack channel, and resonator, respectively. In Figure 10(a), the jet-driven streaming causes negative-value mass fluxes around centreline of the hot buffer and its influence mitigates as the axial position approaches the closed end. We notice non-zero mass fluxes in close proximity to the hot buffer walls which reveals the existence of Rayleigh streaming, i.e., small net drifts driven by boundary-layer effects [45]. In Figure 10(b), the non-zero mass fluxes along the stack channel indicate counter-rotating timeaveraged circular flows from the stack wall surfaces to the centreline. Such inner mass streaming is little known to experimentalists but can be investigated via LES. In Figure 10(c), the Rayleigh streaming is also evident with circulation direction opposite to that in the hot buffer. The time-averaged circulation becomes weaker as the axial position approaches the open end.
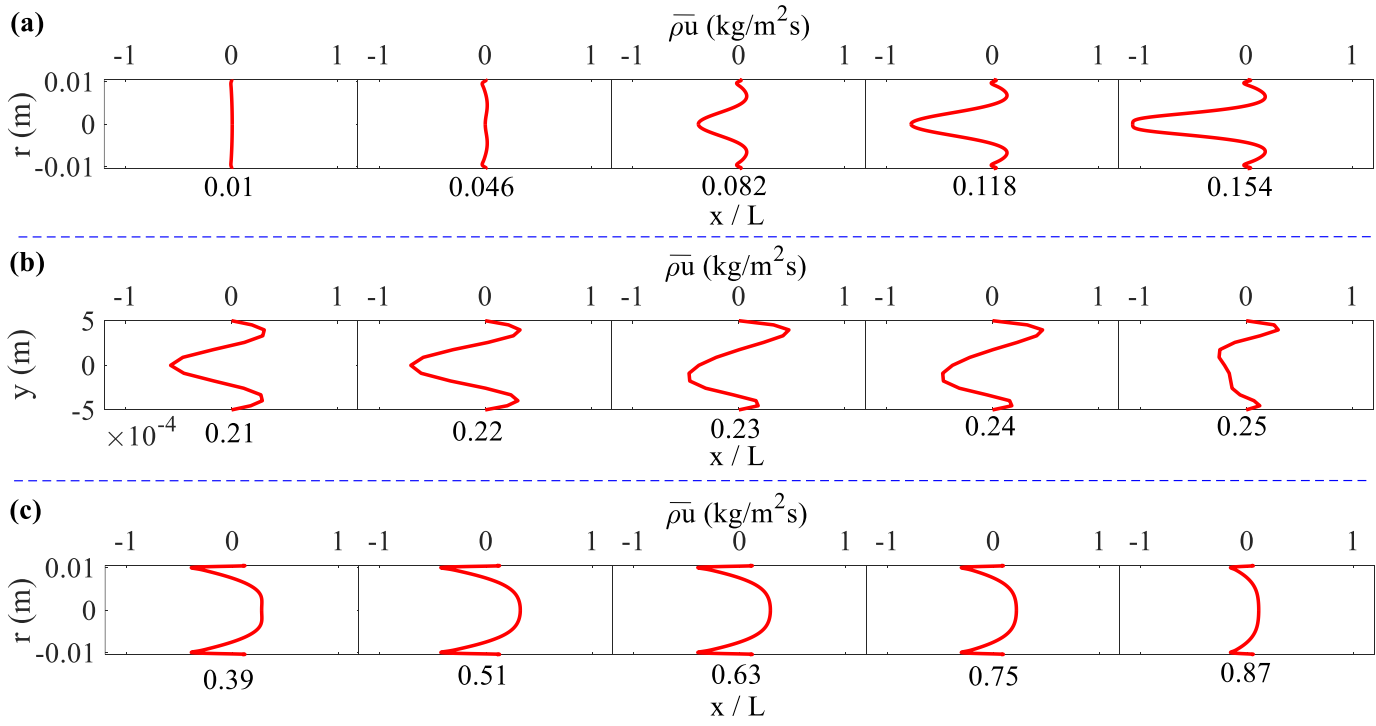

Figure 10. Profiles of time-averaged mass flux $\overline{\rho u}$ along (a) the hot buffer, (b) one representative stack channel and (c) the resonator from LES at $T_{\mathrm{h}}=700 \mathrm{~K}$. 


\section{Heat transfer characteristics}

\subsection{Heat diffusion within boundary layers}

Figure 11 illustrates the instantaneous temperature profiles at the same instants and axial positions as Figure 7 during one cycle at $T_{\mathrm{h}}=700 \mathrm{~K}$. In Figure 11(b), the temperature gradient is zero in the resonator. The phase between the temperature and velocity oscillations outside the thermal boundary layers is around $\pi / 2$. Within the thermal boundary layers, heat conduction shifts the oscillatory temperature slightly in a similar way to that the viscosity does on velocity. Near-wall overshoots in the temperature profiles show up which were also observed in the experiment undertaken by Yu et al [46]. However, in Figure 11(a), the phase is altered significantly in the stack region by the non-zero temperature gradient and deflects from $\pi / 2$. Thus, the space-averaged temperature is not close to zero at $\phi_{3}$ or $\phi_{7}$ when the velocity is at its maximum or minimum.

(a)

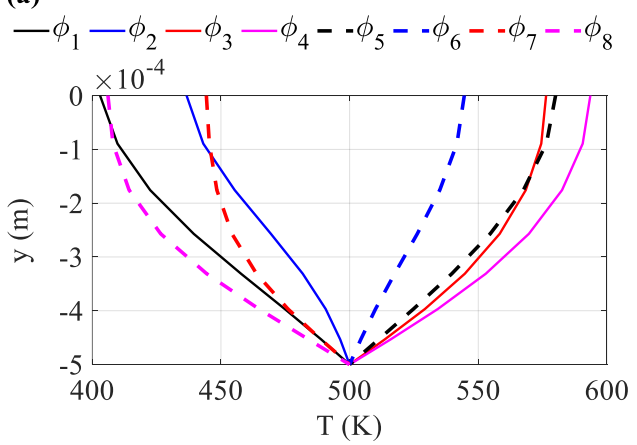

(b) $-\phi_{1}-\phi_{2}-\phi_{3}-\phi_{4}--\phi_{5}--\phi_{6}--\phi_{7}--\phi_{8}$

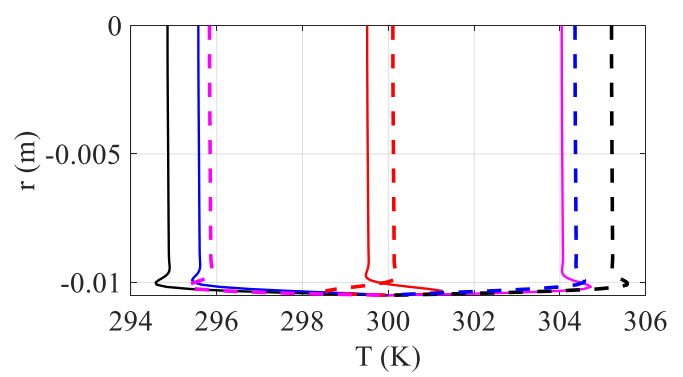

Figure 11. Instantaneous temperature profiles during one acoustic cycle at $T_{\mathrm{h}}=700 \mathrm{~K}$ from LES. (a) Stack channels at $x=0.115 \mathrm{~m}$ where $\tau_{\alpha}=2.25 \times 10^{-3} \mathrm{~s}$. (b) Resonator at $x=0.25 \mathrm{~m}$ where $\tau_{\alpha}=2.47 \mathrm{~s}$.

The energy equation in Equation (5) can be transformed into an entropy wave equation

$$
\frac{\partial s^{\prime}}{\partial t}-\alpha \nabla^{2} s^{\prime}=\frac{\alpha c_{p}}{\rho_{0} a^{2}}(\gamma-1) \nabla^{2} p^{\prime}
$$

Hence, apart from planar acoustic wave, there is diffusive entropy wave in the oscillatory flow field. Similar to $\tau_{v}$, we can use the thermal relaxation time

$$
\tau_{\alpha}=\left(D_{0} / \delta_{\alpha}\right)^{2} / \omega
$$

to characterize the propagation of diffusive entropy wave. $\delta_{\alpha}=\sqrt{2 \kappa / \rho \omega}$ denotes the thermal penetration depth. If $D_{0}$ is small or the heat conductivity $\kappa$ is large, $\tau_{\alpha}$ is small and heat conduction occurs promptly. For example, $\tau_{\alpha}=2.25 \times 10^{-3} \mathrm{~s}$ in the stack channel at $x=0.115 \mathrm{~m}$ in Figure 11(a). In this regard, the diffusive entropy wave dominates, and the walls can be viewed quasi-isothermal. On the contrary, if $D_{0}$ is large or the heat conductivity $\kappa$ is small, $\tau_{\alpha}$ is large and the diffusive effects are small. For instance, 
$\tau_{\alpha}=2.47 \mathrm{~s}$ in the resonator at $x=0.25 \mathrm{~m}$ in Figure 11(b). In this case, the planar acoustic wave dominates, and the walls can be viewed quasi-adiabatic.

\subsection{Heat leaks at the stack ends}

The mass streaming introduced in Section 5.3 affects the mean temperature field at the stack ends. Figure 12(a) presents the time-averaged (mean) temperature contour of the full-scale TAE in the axisymmetric plane from LES at $T_{\mathrm{h}}=700 \mathrm{~K}$. The mass streaming transports colder gas parcels from the stack left end into the hot buffer, and carries hot gas parcels from the stack right end into the resonator, as shown in the enlarged inset in Figure 12(b). Kuzuu and Hasegawa [17] also observed similar heat leak phenomenon in their 2-D numerical study. Generally, heat leaks at the stack ends are undesirable since they reduce the amount of heat utilized for sound generation and thereby decrease the thermal efficiency of the TAE.

(a)

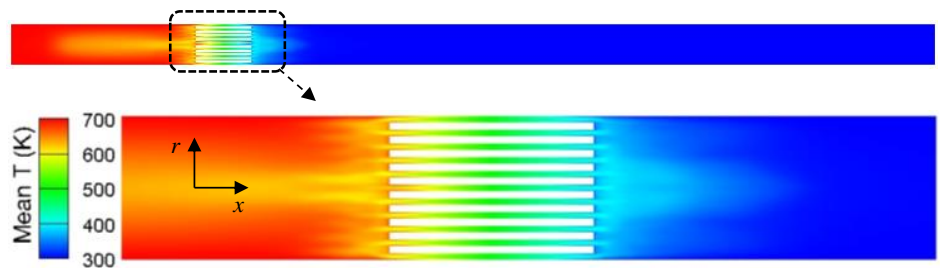

Figure 12. Contours of the time-averaged temperature in the axisymmetric plane from LES at $T_{\mathrm{h}}=$ 700 K. (a) Full-scale TAE; (b) close-up view of the stack region.

\subsection{Heat fluxes in the transversal direction}

Section 6.1 indicates that there are heat fluxes in the transversal direction at the solid surfaces due to heat diffusion. The time-averaged transversal heat flux $q_{t}$ is therefore

$$
q_{t}=-\left.\kappa \frac{\partial T_{m}}{\partial n}\right|_{\text {surface }}=h\left(T_{w}-\left\langle T_{m}\right\rangle\right)
$$

where $n$ represents the direction normal to the wall surfaces. $h$ is the heat transfer coefficient that relies on $q_{t}$ and the difference between the wall temperature $T_{w}$ and space-averaged mean temperature $\left\langle T_{m}\right\rangle$ of the fluid. Figure 13 presents $q_{t}$ along the TAE from LES at $T_{\mathrm{h}}=700 \mathrm{~K}$. Obviously, the right-end part of the hot buffer and left half of the stack serve as the "hot heat exchangers" which supply heat to the working fluid, while the right half of the stack and left-end part of resonator act as the "ambient heat exchangers", extracting heat from the fluid. The close-up view around the stack region in Figure 13 shows the differences in $q_{t}$ of different stack plate surfaces. Since there are no external loads attached to the TAE ends, conservation of energy indicates that the total heat supplied equals the total heat extracted. 


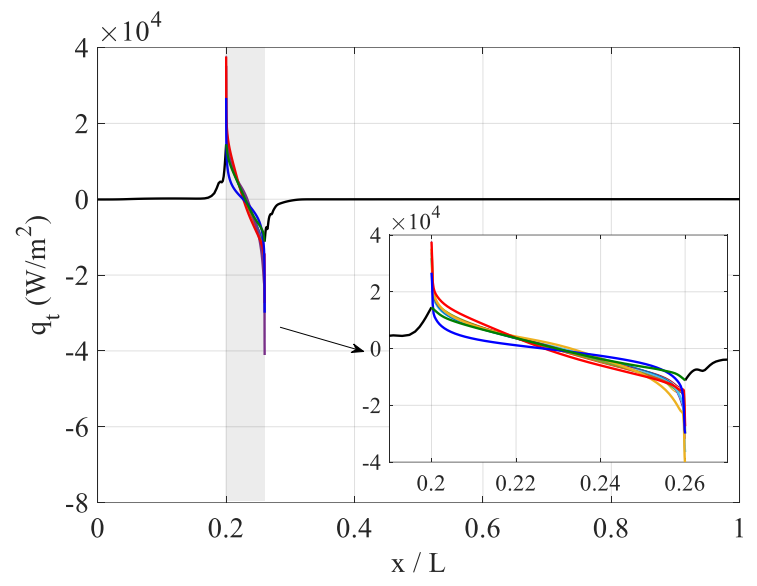

Figure 13. Time-averaged heat flux distribution along the tube from LES at $T_{\mathrm{h}}=700 \mathrm{~K}$.

In the stack region, the transversal heat flow absorbed and extracted can be estimated by

$$
\begin{gathered}
Q_{t, \text { in }}=\sum \int_{0.2}^{0.23} q_{t, s i} \Pi_{s i} d(x / L) \\
Q_{t, \text { out }}=\sum \int_{0.23}^{0.26} q_{t, s i} \Pi_{s i} d(x / L)
\end{gathered}
$$

where $q_{t, s i}$ and $\Pi_{s i}$ are the transversal heat fluxes and perimeters of different stack plates. Therefore, first law of thermodynamics gives

$$
\Delta W_{x}=Q_{t, \text { in }}-\left|Q_{t, \text { out }}\right|
$$

where $\Delta W_{x}$ stands for the acoustic power generated in the stack. Figure 14 shows the effect of $T_{\mathrm{h}}$ on $Q_{t, i n}, Q_{t, o u t}$ and $\Delta W_{x}$. It is evident that $Q_{t, \text { in }}$ and $\left|Q_{t, o u t}\right|$ increase because the oscillation amplitude increases. The increase of $\Delta W_{x}$ demonstrates larger viscous and thermal losses outside the stack region.

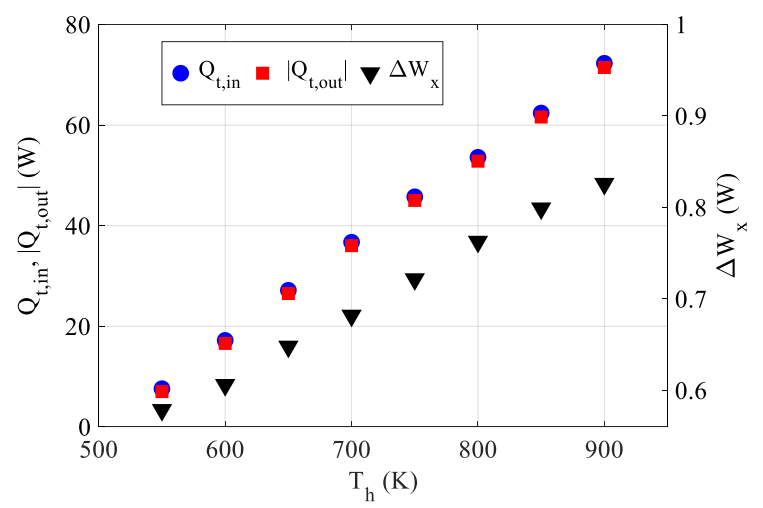

Figure 14. Effect of temperature difference on the energy conversion in the stack region. 


\section{Conclusions}

With the support of high-performance computing (HPC), high-fidelity three-dimensional (3-D) large eddy simulation (LES) of a full-scale quarter-wavelength standing-wave thermoacoustic engine (TAE) has been conducted in this study. Sensitivity of the steady-state responses to the grid and time-step sizes is first investigated. Then, the dynamic, acoustic, hydrodynamic and heat transfer characteristics of the thermoacoustic engine are numerically studied by resolving the fully compressible Navier-Stokes equations on high-resolution structured grids. This paper facilitates the comprehension of thermally induced oscillatory flow inside the thermoacoustic engine and its overall performance from broader viewpoints. The large eddy simulation not only sheds light on the mechanisms responsible for the acoustic power generation inside the stack, but also gives insight into various nonlinear phenomena that are difficult to examine through experiments. We make the following conclusions from this study:

(1) In the dynamic analysis, instead of solving the inhomogeneous acoustic wave directly, a systemwide reduced-order network model based on the linearized equations is utilized to study the dynamic behaviour of acoustic pressure in the initial start-up process. Theoretical calculations are compared with numerical estimates from both large eddy simulations and unsteady ReynoldsAveraged Navier-Stokes (URANS) simulations. The large eddy simulation outperforms unsteady Reynolds-Averaged Navier-Stokes simulation at predicting the oscillation frequency and growth rate at different hot-end temperatures.

(2) In the acoustic analysis, Fourier Series Model is employed to reveal the predominant unstable longitudinal modes excited inside the thermoacoustic engine. Analysis on acoustic power further indicates that the stack serves as an energy source for the fundamental mode while it extracts acoustic power from the second harmonic.

(3) In the hydrodynamic analysis, examination of the velocity profiles indicates that there is diffusive vorticity wave in the oscillatory flow field which dissipates momentum irreversibly at a time scale of $\tau_{v}$. Vortex shedding around the stack ends that contributes to minor losses in the thermoacoustic engine is visualized by three-dimensional representations of vortex core regions and velocity streamlines. Jet-driven streaming around the stack ends, inner steaming inside the stack channels, and Rayleigh streaming in the vicinity of tube walls are observed with non-zero mass fluxes at different axial positions along the thermoacoustic engine.

(4) In the heat transfer analysis, examination of the temperature profiles indicates that there is diffusive entropy wave in the oscillatory flow field which diffuses heat irreversibly at a time scale of $\tau_{\alpha}$. Heat leaks caused by mass streaming are found at the ends of the stack. The positive and negative transversal heat fluxes at the solid surfaces demonstrate heat addition to and extraction from the working fluid. In the stack region, the generation of acoustic power shows strong dependence on the hot-end temperature. 
The large eddy simulation methodology in this research proves effective in studying the performance of the standing-wave thermoacoustic engine both quantitively and qualitatively. Prospective work may involve the application of this numerical approach to simulate the thermally induced oscillatory flow in more complicated travelling-wave thermoacoustic systems.

\section{Acknowledgements}

The authors want to acknowledge the high-performance computing (HPC) provided by New Zealand eScience Infrastructure (NeSI). The authors are grateful for the Universitas 21 Doctoral Student Mobility Scholarship from the University of Auckland and financial support from China Scholarship Council (no. 201608630046).

\section{Appendix A. Thermal boundary condition}

Figure A1 illustrates a piece of stack plate sandwiched between a pair of hot and ambient heat exchangers (HEs) in the axisymmetric plane. In this study, the thermal boundary condition for the stack is expressed as

$$
\left\{\begin{array}{l}
\mathrm{AB}: T_{w}(x)=T_{h} \\
\mathrm{AC} \text { or } \mathrm{BD}: T_{w}(x)=\left(T_{h}-T_{a}\right)\left(x-x_{l}\right) /\left(x_{l}-x_{r}\right)+T_{h} \\
\mathrm{CD}: T_{w}(x)=T_{a}
\end{array}\right.
$$

where $T_{w}$ is the solid surface temperature. Surfaces AB and CD locate at $x=x_{l}$ and $x=x_{r}$, respectively. We choose such thermal boundary condition due to the following two reasons.

(i) Computational cost. There is no need to mesh solid domains of stack plates if the stack surface temperatures are specified. This would reduce the numbers of nodes in computation and avoid the calculation of solid temperature within the plates, which is of no interest in present research.

(ii) Comparison with the network model. The surface temperatures on $\mathrm{AC}$ and $\mathrm{BD}$ are linear according to Equation (A1). They are consistent with the assumptions made in the network model.

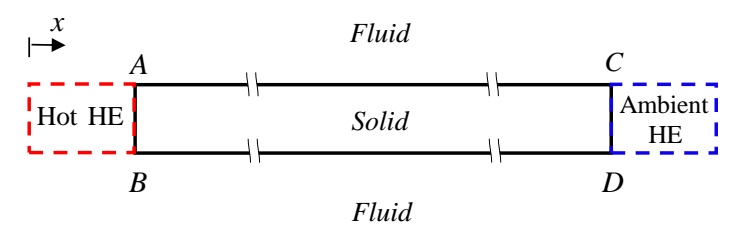

Figure A1. Axisymmetric-plane view of a piece of stack plate sandwiched between a pair of hot and ambient heat exchangers (HEs).

\section{Appendix B. Experimental proof of network model}

The reduced-order network model has been validated in our previous experiments on thermoacoustic devices of similar configurations and sizes $[35,36]$. Figure B1 displays the time history of closed-end 
acoustic pressure $p$ inside a thermoacoustic-piezoelectric energy harvester. See Ref. [35] for detailed descriptions of the experimental procedure and measurements. Note that the impedances $Z(0)$ and $Z(L)$ in the network model in Ref. [35] are equivalent acoustic impedances of the piezoelectric transducer and acoustic radiation. A short period (from $t=11 \mathrm{~s}$ to $12 \mathrm{~s}$ ) of $p$ in the initial start-up process is postprocessed (by Fast Fourier Transform and plotting $\ln \left(p_{\text {peak }}\right)$ versus $t$, where $p_{\text {peak }}$ is the peak value of $p$ ) to obtain the oscillation frequency and growth rate in the experiment. Theoretical predictions (dashed lines) from the network model are also displayed for comparison. The theoretical estimates coincide with the experimental measurements which credits the network method in predicting the dynamic behaviour of a thermoacoustic device.

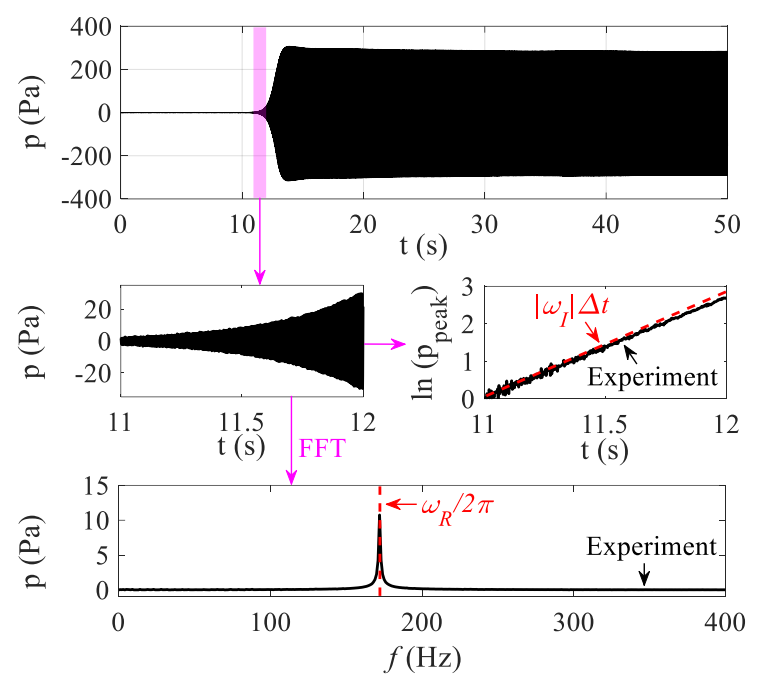

Figure B1. Experimental proof of the reduced-order network model. Data are reproduced from Ref. [35] with the permission of Elsevier Ltd. Dashed lines are theoretical predictions using the network method.

\section{References}

[1] Swift GW. Thermoacoustic engines. The Journal of the Acoustical Society of America. 1988;84:1145-80.

[2] Yu Z, Jaworski AJ, Backhaus S. Travelling-wave thermoacoustic electricity generator using an ultra-compliant alternator for utilization of low-grade thermal energy. Applied Energy. 2012;99:13545.

[3] Wang K, Sun D, Zhang J, Xu Y, Zou J, Wu K, et al. Operating characteristics and performance improvements of a 500W traveling-wave thermoacoustic electric generator. Applied Energy. 2015;160:853-62.

[4] Tsuda K, Ueda Y. Critical temperature of traveling-and standing-wave thermoacoustic engines using a wet regenerator. Applied energy. 2017;196:62-7. 
[5] Timmer MA, de Blok K, van der Meer TH. Review on the conversion of thermoacoustic power into electricity. The Journal of the Acoustical Society of America. 2018;143:841-57.

[6] Jin T, Yang R, Wang Y, Liu Y, Feng Y. Phase adjustment analysis and performance of a looped thermoacoustic prime mover with compliance/resistance tube. Applied Energy. 2016;183:290-8.

[7] Tan J, Wei J, Jin T. Electrical-analogy network model of a modified two-phase thermofluidic oscillator with regenerator for low-grade heat recovery. Applied Energy. 2020;262:114539.

[8] Xu J, Luo E, Hochgreb S. Study on a heat-driven thermoacoustic refrigerator for low-grade heat recovery. Applied Energy. 2020;271:115167.

[9] Shen C, He Y, Li Y, Ke H, Zhang D, Liu Y. Performance of solar powered thermoacoustic engine at different tilted angles. Applied Thermal Engineering. 2009;29:2745-56.

[10] Zhao D, Li S, Yang W, Zhang Z. Numerical investigation of the effect of distributed heat sources on heat-to-sound conversion in a T-shaped thermoacoustic system. Applied Energy. 2015;144:204-13. [11] Rayleigh JWSB. The theory of sound: Macmillan; 1896.

[12] Rott N. Thermoacoustics. Advances in applied mechanics. 1980;20:135-75.

[13] Swift GW. Thermoacoustics: A unifying perspective for some engines and refrigerators: Springer; 2017.

[14] Hao H, Scalo C, Sen M, Semperlotti F. Thermoacoustics of solids: A pathway to solid state engines and refrigerators. Journal of Applied Physics. 2018;123:024903.

[15] Hao H, Scalo C, Semperlotti F. Traveling and standing thermoacoustic waves in solid media. Journal of Sound and Vibration. 2019;449:30-42.

[16] Swift G. Analysis and performance of a large thermoacoustic engine. The Journal of the Acoustical Society of America. 1992;92:1551-63.

[17] Kuzuu K, Hasegawa S. Effect of non-linear flow behavior on heat transfer in a thermoacoustic engine core. International Journal of Heat and Mass Transfer. 2017;108:1591-601.

[18] El-Rahman AIA, Abdelfattah WA, Fouad MA. A 3D investigation of thermoacoustic fields in a square stack. International Journal of Heat and Mass Transfer. 2017;108:292-300.

[19] Zink F, Vipperman J, Schaefer L. CFD simulation of a thermoacoustic engine with coiled resonator. International Communications in Heat and Mass Transfer. 2010;37:226-9.

[20] Chen G, Krishan G, Yang Y, Tang L, Mace B. Numerical investigation of synthetic jets driven by thermoacoustic standing waves. International Journal of Heat and Mass Transfer. 2020;146:118859.

[21] Chen G, Tang L, Mace BR. Bistability and triggering in a thermoacoustic engine: A numerical study. International Journal of Heat and Mass Transfer. 2020;157:119951.

[22] Mergen S, Yıldırım E, Turkoglu H. Numerical study on effects of computational domain length on flow field in standing wave thermoacoustic couple. Cryogenics. 2019;98:139-47.

[23] Skaria M, Rasheed KA, Shafi K, Kasthurirengan S, Behera U. Simulation studies on the performance of thermoacoustic prime movers and refrigerator. Computers \& Fluids. 2015;111:127-36. 
[24] Ilori OM, Jaworski AJ, Mao X. Experimental and numerical investigations of thermal characteristics of heat exchangers in oscillatory flow. Applied Thermal Engineering. 2018;144:910-25. [25] Pope SB. Turbulent flows. IOP Publishing; 2001.

[26] Chen G, Tang L, Mace BR. Theoretical and experimental investigation of the dynamic behaviour of a standing-wave thermoacoustic engine with various boundary conditions. International Journal of Heat and Mass Transfer. 2018;123:367-81.

[27] Chen G, Tang L, Yu Z. Underlying physics of limit-cycle, beating and quasi-periodic oscillations in thermoacoustic devices. Journal of Physics D: Applied Physics. 2020;53:215502.

[28] Zink F, Vipperman J, Schaefer L. CFD simulation of thermoacoustic cooling. International journal of heat and mass transfer. 2010;53:3940-6.

[29] Sutherland W. LII. The viscosity of gases and molecular force. The London, Edinburgh, and Dublin Philosophical Magazine and Journal of Science. 1893;36:507-31.

[30] Nicoud F, Ducros F. Subgrid-scale stress modelling based on the square of the velocity gradient tensor. Flow, turbulence and Combustion. 1999;62:183-200.

[31] Anderson JD, Wendt J. Computational fluid dynamics: Springer; 1995.

[32] Wang K, Sun DM, Zhang J, Zou J, Wu K, Qiu LM, et al. Numerical simulation on onset characteristics of traveling-wave thermoacoustic engines based on a time-domain network model. International Journal of Thermal Sciences. 2015;94:61-71.

[33] Ueda Y, Kato C. Stability analysis of thermally induced spontaneous gas oscillations in straight and looped tubes. The Journal of the Acoustical Society of America. 2008;124:851-8.

[34] Guedra M, Penelet G. On the use of a complex frequency for the description of thermoacoustic engines. Acta Acustica united with Acustica. 2012;98:232-41.

[35] Chen G, Tang L, Mace BR. Modelling and analysis of a thermoacoustic-piezoelectric energy harvester. Applied Thermal Engineering. 2019;150:532-44.

[36] Chen G, Tang L, Yang Z, Tao K, Yu Z. An electret - based thermoacoustic - electrostatic power generator. International Journal of Energy Research. 2020;44:2298-305.

[37] Arnott WP, Bass HE, Raspet R. General formulation of thermoacoustics for stacks having arbitrarily shaped pore cross sections. The Journal of the Acoustical Society of America. 1991;90:322837.

[38] Biwa T, Yazaki T. Observation of energy cascade creating periodic shock waves in a resonator. The Journal of the Acoustical Society of America. 2010;127:1189-92.

[39] Shi L, Yu Z, Jaworski AJ. Application of laser-based instrumentation for measurement of timeresolved temperature and velocity fields in the thermoacoustic system. International Journal of Thermal Sciences. 2010;49:1688-701.

[40] Mao X, Yu Z, Jaworski AJ, Marx D. PIV studies of coherent structures generated at the end of a stack of parallel plates in a standing wave acoustic field. Experiments in Fluids. 2008;45:833-46. 
[41] Gedeon D. DC gas flows in Stirling and pulse-tube cryocoolers. Cryocoolers. 1997;9:385-92.

[42] Matveev K, Backhaus S, Swift GW. The influence of gravity on Rayleigh streaming in thermoacoustic systems. The Journal of the Acoustical Society of America. 2004;115:2380-1.

[43] Boluriaan S, Morris PJ. Acoustic streaming: from Rayleigh to today. International Journal of aeroacoustics. 2003;2:255-92.

[44] Kirkconnell C. Experimental investigation of a unique pulse tube expander design. Cryocoolers 10: Springer; 2002. p. 239-47.

[45] Olson J, Swift G. Acoustic streaming in pulse tube refrigerators: tapered pulse tubes. Cryogenics. 1997;37:769-76.

[46] Yu Z, Mao X, Jaworski AJ. Experimental study of heat transfer in oscillatory gas flow inside a parallel-plate channel with imposed axial temperature gradient. International Journal of Heat and Mass Transfer. 2014;77:1023-32. 